DRAFT VERSION OCTOBER 10, 2017

Preprint typeset using LATEX style AASTeX6 v. 1.0

\title{
CLOUDLESS ATMOSPHERES FOR YOUNG LOW-GRAVITY SUBSTELLAR OBJECTS
}

\author{
P. Tremblin ${ }^{1}$ and G. Chabrier ${ }^{2,3}$ and I. Baraffe ${ }^{2,3}$ and Michael. C. Liu ${ }^{4,5}$ and E. A. Magnier ${ }^{4}$ and P.-O. Lagage, ${ }^{6,7}$ and C. Alves de Oliveira ${ }^{8}$ \\ and A. J. Burgasser ${ }^{9}$ and D. S. Amundsen ${ }^{10,11}$ and B. Drummond ${ }^{2}$
}

\footnotetext{
${ }^{1}$ Maison de la Simulation, CEA, CNRS, Univ. Paris-Sud, UVSQ, Université Paris-Saclay, 91191 Gif-sur-Yvette, France

2 Astrophysics Group, University of Exeter, EX4 4QL Exeter, UK

${ }^{3}$ Ecole Normale Supérieure de Lyon, CRAL, UMR CNRS 5574, 69364 Lyon Cedex 07, France

${ }^{4}$ Institute for Astronomy, University of Hawaii, 2680 Woodlawn Drive, Honolulu, HI 96822, USA

${ }^{5}$ Visiting Astronomer at the Infrared Telescope Facility, which is operated by the University of Hawaii under Cooperative Agreement no. NNX-08AE38A with the National Aeronautics and Space Administration, Science Mission Directorate, Planetary Astronomy Program.

${ }^{6}$ Irfu, CEA, Université Paris-Saclay, F-9119 Gif-sur Yvette, France

${ }^{7}$ Université Paris Diderot, AIM, Sorbonne Paris Cité, CEA, CNRS, F-91191 Gif-sur-Yvette, France

${ }^{8}$ European Space Agency, c/o STScI, 3700 San Martin Drive, Baltimore, MD 21218, USA

${ }^{9}$ UC San Diego, M/C 0424, 9500 Gilman Drive, La Jolla, CA 92093, USA

${ }^{10}$ Department of Applied Physics and Applied Mathematics, Columbia University, New York, NY 10025, USA

${ }^{11}$ NASA Goddard Institute for Space Studies, New York, NY 10025, USA
}

\begin{abstract}
Atmospheric modeling of low-gravity (VL-G) young brown dwarfs remains a challenge. The presence of very thick clouds has been suggested because of their extremely red near-infrared (NIR) spectra, but no cloud models provide a good fit to the data with a radius compatible with evolutionary models for these objects. We show that cloudless atmospheres assuming a temperature gradient reduction caused by fingering convection provides a very good model to match the observed VL-G NIR spectra. The sequence of extremely red colors in the NIR for atmospheres with effective temperature from $\sim 2000 \mathrm{~K}$ down to $\sim 1200 \mathrm{~K}$ is very well reproduced with predicted radii typical of young low-gravity objects. Future observations with NIRSPEC and MIRI on the James Webb Space Telescope (JWST) will provide more constrains in the mid-infrared, helping to confirm/refute whether or not the NIR reddening is caused by fingering convection. We suggest that the presence/absence of clouds will be directly determined by the silicate absorption features that can be observed with MIRI. JWST will therefore be able to better characterize the atmosphere of these hot young brown dwarfs and their low-gravity exoplanet analogues.
\end{abstract}

Keywords: Methods: observational — Methods: numerical — brown dwarfs — planets and satellites: atmospheres

\section{INTRODUCTION}

Although representing a small fraction of the entire brown dwarf population, young brown dwarfs $(\leq 150$ Myrs) largely populate and even dominate the redward part $(\mathrm{J}-\mathrm{K} \gtrsim 0.8)$ of the color-magnitude diagram (e.g. Chabrier 2002) and are of great interest because of their large radii and low surface gravity. Their study provides a promising approach to better understand low-gravity ultracool analog atmospheres such as those of young gas giant exoplanets. Recently, widefield surveys have led to the identification of large samples of such low-gravity objects (e.g. Gagné et al. 2015a; Aller et al. 2016). This population forms a separate sequence in near-

pascal.tremblin@cea.fr infrared (NIR) color-magnitude diagrams (CMD) from the field objects (Liu et al. 2013, 2016; Faherty et al. 2016). This sequence is typically $\sim 0.5$ mag redder in $J-K$ color and has been interpreted as the presence of thicker clouds with small particles, even though current cloud models struggle to fit these data (e.g. Liu et al. 2016). The models of Saumon and Marley (2008); Marley et al. (2012) cannot reach the reddest colors at a given magnitude although they have provided reasonable fits and radii for the HR8799 planets (Marley et al. 2012) and BT-Settl models lead to implausibly small fitted radii (e.g. Liu et al. 2013).

Recently, Tremblin et al. (2016) have proposed that the reddening of NIR colors in standard field L dwarfs and exoplanet analogs such as HR8799c is induced by the reduction of the temperature gradient in their atmospheres. The development of fingering convection caused by the mean molec- 
ular weight gradient implied by the chemical transition between $\mathrm{CO}$ and $\mathrm{CH}_{4}$ could be at the origin of such a temperature gradient reduction. This mechanism naturally explains why the disappearance of the reddening at the $\mathrm{L} / \mathrm{T}$ transition is concomitant with the transition between $\mathrm{CO}$ and $\mathrm{CH}_{4}$ in the atmosphere of these objects.

In this paper, we show that the same model based on Tremblin et al. (2016) reproduce very well spectra of VL-G objects (DENIS J1425-36, 2MASS J2208+29, and PSO J318-22, chosen as late-L members of well-established young moving groups AB Doradus and Beta Pictoris) as well as the sequence in $\mathrm{M}_{J}$ versus $J-K \mathrm{CMD}$. Furthermore, the radii inferred from the spectral fits are in good agreement with evolutionary models of young objects. The derived ages of DENIS J1425-36 and PSO J318-22 are in good agreement with their ages estimated from their membership of their respective moving groups. Therefore, these results support the $\mathrm{CO} / \mathrm{CH}_{4}$ fingering-unstable interpretation of the $\mathrm{L} / \mathrm{T}$ transition of standard field objects and VL-G objects.

\section{SPECTRAL MODELS}

The spectral models are all done with the ATMO code (Amundsen et al. 2014; Tremblin et al. 2015; Drummond et al. 2016). We kept the same opacity sources as in Tremblin et al. (2016) for direct comparison $\left(\mathrm{H}_{2}-\mathrm{H}_{2}, \mathrm{H}_{2}-\mathrm{He}, \mathrm{H}_{2} \mathrm{O}\right.$, $\mathrm{CO}, \mathrm{CO}_{2}, \mathrm{CH}_{4}, \mathrm{NH}_{3}, \mathrm{~K}, \mathrm{Na}$, TiO, VO, and $\mathrm{FeH}$, from the high temperature ExoMol (Tennyson and Yurchenko 2012) and HITEMP (Rothman et al. 2010) line list databases when available).

Similarly to Tremblin et al. (2015, 2016); Drummond et al. (2016), we use the coupling of the radiative/convective code with the CHNO-based chemical network of Venot et al. (2012) for the treatment of the departure from chemical equilibrium in the nitrogen and carbon chemistry. Starting from a converged pressure/temperature (PT) structure at chemical equilibrium, we integrate the time evolution of the abundances of all the chemical species in all the layers of the atmosphere. When turbulent mixing is faster than the chemical timescales (mixing coefficient $\mathrm{K}_{\mathrm{zz}}{ }^{1}$ ), the abundances of some molecules are not at chemical equilibrium anymore and can be controlled by the turbulent diffusion from the deepest layers. The non-equilibrium abundances are computed by solving the time evolution of the continuity equation (see Eq. 10 in Drummond et al. 2016) with the $\operatorname{LSODE}^{2}$ integrator for stiff ordinary differential equations (Hindmarsh 1983). We re-converge the PT structure with the radiative/convective code regularly during the time integration until we reach a steady state for both the chemistry and energy conservation/hydrostatic equilibrium. We then get a co-

\footnotetext{
${ }^{1} \mathrm{~K}_{\mathrm{zZ}}$ is a free parameter and represents the vertical eddy diffusion coefficient

${ }^{2}$ https://computation.llnl.gov/casc/odepack/
}

herent atmospheric structure with out-of-equilibrium chemical abundances. As in Tremblin et al. (2016), we propose that a process similar to fingering convection is responsible for this turbulent mixing and leads to the out-of-equilibrium abundances. Fingering convection can be triggered in the atmospheres of brown dwarfs and exoplanets because of the gradient of mean molecular weight induced by the chemical transitions $\mathrm{CO} / \mathrm{CH}_{4}$ and $\mathrm{N}_{2} / \mathrm{NH}_{3}$ at chemical equilibrium. Similarly to convective motions that lead to an adiabatic PT structure in the deep atmosphere, fingering convection could impact the PT structure of the atmosphere. As in Tremblin et al. (2016), we propose that fingering-convective motions can induce a temperature gradient reduction leading to a reddening in the modeled spectrum. This temperature gradient reduction in the atmosphere is modelled using an artificially reduced adiabatic index $\gamma$ in between two pressure levels on top of the convective zone.

In Fig. 1, we show the influence of various model parameters at an effective temperature of $1700 \mathrm{~K}$ and $1300 \mathrm{~K}$, representative of the objects studied in this letter. At $1700 \mathrm{~K}$ we can see a degeneracy between gravity and metallicity on the spectral shape of the $H$ and $K$ bands. Decreasing gravity or increasing metallicity tends to form a triangular $H$ band and a strong and flatter $K$ band. Decreasing the temperature gradient in the atmosphere by decreasing the effective adiabatic index $\gamma$, increases the reddening in $J-K$. Increasing the surface gravity at an effective temperature of $1300 \mathrm{~K}$ has a strong effect on out-of-equilibrium chemistry and the quenching of $\mathrm{CO}$ in the deep atmosphere. At higher gravities, the $\mathrm{CO} / \mathrm{CH}_{4}$ transition is deeper in the atmosphere, hence it is more difficult to prevent the formation of $\mathrm{CH}_{4}$ (strongly appearing at $\log g=4.5$ ) for a given mixing coefficient $\mathrm{K}_{\mathrm{zz}}$. Thus varying $\mathrm{K}_{\mathrm{zz}}$ would approximately lead to the same effect as varying gravity for $\mathrm{T}_{\mathrm{eff}}=1300 \mathrm{~K}$. For the fitting of the observations we have probed a similar parameter space $\log g$ in $[3.5,4.5]$, $[\mathrm{M} / \mathrm{H}]$ in $[0,0.5], \mathrm{T}_{\mathrm{eff}}$ in $[1200,1800]$, and $\gamma$ in $[1.01,1.1]$.

\subsection{DENIS J1425279-365023}

DENIS J1425279-365023 was first identified as an L5 dwarf (Kendall et al. 2004) with a trigonometric distance of $\sim 11.57 \pm 0.11$ pc (Dieterich et al. 2014). A SpeX Prism obtained by Gagné et al. (2015a) indicates that the object is a L4INT-G object based on the index-based classification of Allers and Liu (2013). Based on the galactic position and space velocity of the object, Gagné et al. (2015b,a) have also concluded that DENIS J1425-36 is a bona field member of the AB Doradus Moving Group $\left(149_{-19}^{+51}\right.$ Myrs, Bell et al. 2015).

Figure 2 shows the SpeX prism spectrum of DENIS J142536 compared to a model obtained with the ATMO code. As in Tremblin et al. (2016), the reddening of the modeled spectrum is obtained by a temperature gradient reduction in the atmosphere using an artificially reduced adiabatic index $\gamma$. Tremblin et al. (2016) have obtained a very good fit to field 

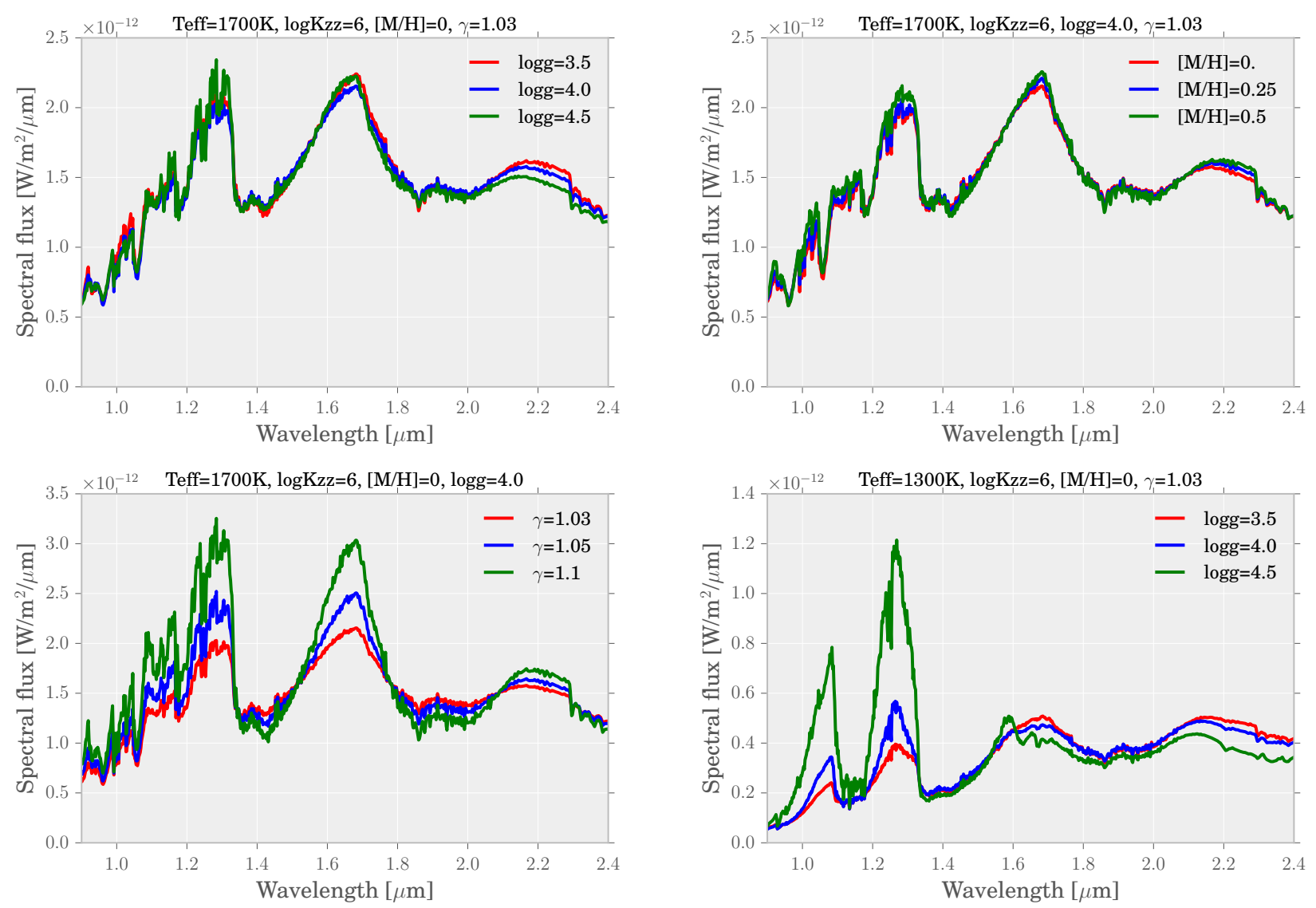

Figure 1. Effects of varying different parameters on the spectral models obtained with the ATMO code.

standard $\mathrm{L}$ dwarfs with a $\gamma$ value around 1.05 in the region between $\sim 100$ bars and $\sim 2$ bars. This result has recently been confirmed by Burningham et al. (2017) although the authors suggest that sophisticated cloud models could provide a slightly better fit. For DENIS J1425-36, we show that the same model provides a good fit to the observed spectrum with a shallower temperature gradient with $\gamma \approx 1.02$ extending up to 0.25 bars in the atmosphere. As anticipated from the $H$ and $K$ band spectral shapes, we also find a low surface gravity, which indicates the youth of the object but also a slightly supersolar metallicity. Most importantly, we have obtained a radius of $\sim 0.12 \mathrm{R}_{\odot}$ for DENIS J1425-36 which corresponds to an age of 100-150 Myrs, consistent with evolutionary models (Baraffe et al. 2003) and the age of the AB Doradus Moving Group.

\section{2. $2 M A S S W J 2208136+292121$}

2MASSW J2208136+292121 was first discovered by Kirkpatrick et al. (2000) and classified as a peculiar L2. The peculiarity was later understood as signs of low gravity and the object is now classified as a L3VL-G with a parallax distance of $47.6 \pm 2$ pc (Liu et al. 2016). Based on its optical spectrum, Kirkpatrick et al. (2008) estimated an age of $\sim 100$ Myr for the object. Gagné et al. (2014) has estimated a probability of only $10 \%$ for a potential membership to the Beta
Pictoris Moving Group, which would suggest a younger age of 24 \pm 3 Myrs (Bell et al. 2015).

Figure 2 shows the SpeX prism spectrum of 2MASS J2208+29 compared to a model obtained with the ATMO code. This object appears to have a CMD position discrepant with its gravity classification (Liu et al. 2016) with a shift of only 0.1-0.2 mag in $J-K$ relative to the standard field objects. This fact directly reflects in the effective $\gamma$ value of 1.05 found with the spectral model, a value closer to the one used for standard field object (Tremblin et al. 2016). The shape of the spectrum, e.g. the very peaked $H$ band, clearly indicates a low gravity and/or a high metallicity for the object, as found with our model. We have found a radius similar to the one of DENIS J1425-36, around $0.12 \mathrm{R}_{\odot}$. This radius corresponds to an age of 100-150 Myrs with evolutionnary models. This result confirms the "optical" age found by Kirkpatrick et al. (2008) and argue against a membership to the Beta Pictoris Moving Group.

\subsection{PSO J318.5338-22.8603}

PSO J318.5338-22.8603 has been identified as the reddest field object ever observed (Liu et al. 2013) with a parallax distance of $24.6 \pm 1.1 \mathrm{pc}$. Its $J-K$ color is $2.78 \mathrm{mag}$, which has been interpreted as the sign of an unusually dusty atmosphere. This interpretation, however, is weakened by the dif- 

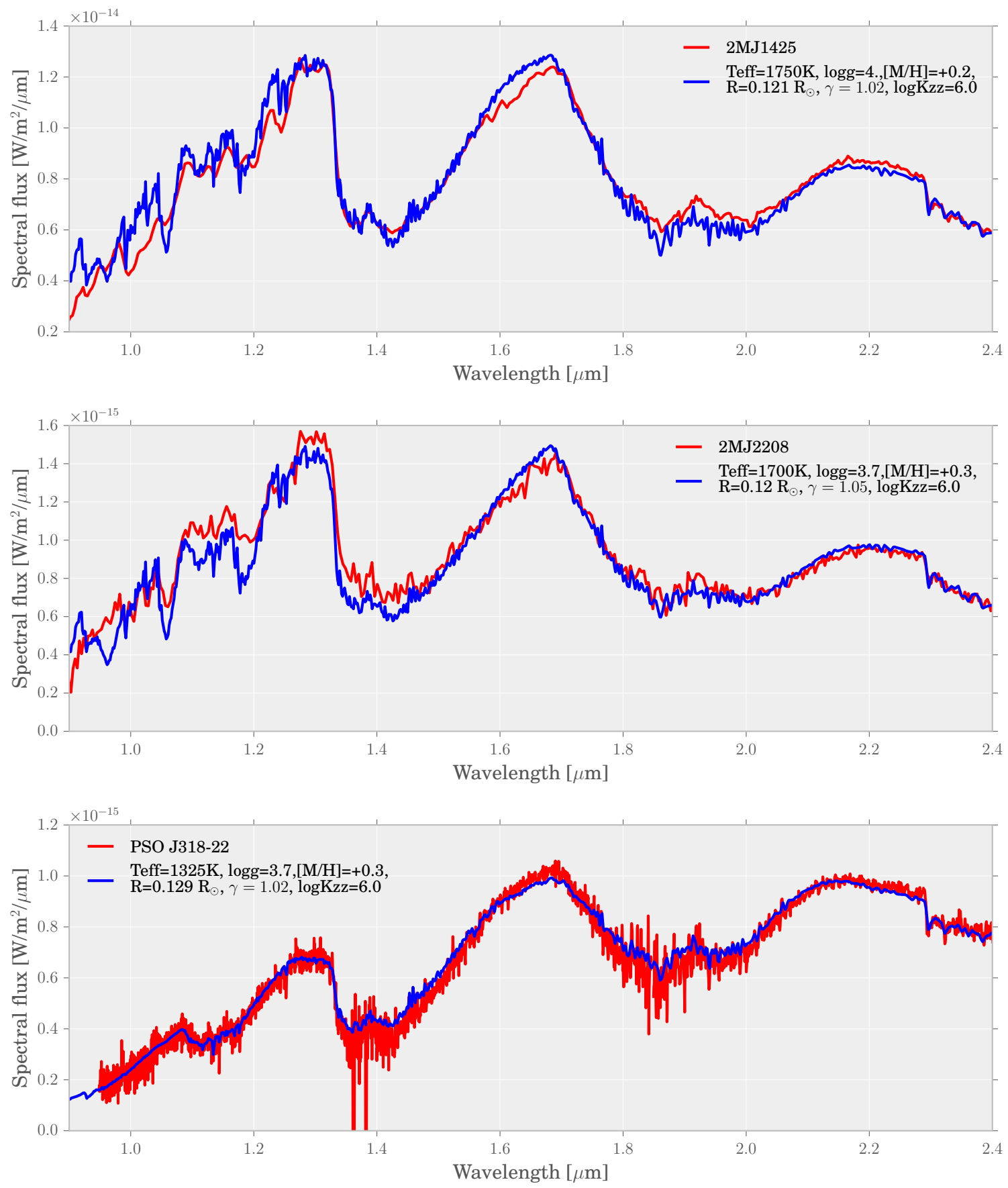

Figure 2. Spectral models obtained with the ATMO code using a temperature gradient reduction in the atmosphere (Tremblin et al. 2016) compared with the SpeX prism spectra of DENIS J1425279-365023 (Bardalez Gagliuffi et al. 2014) and 2MASSW J2208136+292121 (Allers and Liu 2013), and the GNIRS spectrum of PSO J318.5338-22.8603 (Liu et al. 2013).

ficulty of fitting the NIR spectrum with cloud models. A good fit can be obtained with BT-Settl models (Liu et al. 2013) but with an implausibly small radius (around $0.08 \mathbf{R}_{\odot}$ ) for such a young object. Its membership to the Beta Pictoris Moving Group suggests an age around 24 \pm 3 Myrs (Bell et al. 2015).
Figure 2 shows the GNIRS ${ }^{3}$ spectrum of PSO J318-22 compared to a model obtained with the ATMO code. The correspondence between the model and the observed spectrum is remarkably good and emphasizes the high precision of the $\mathrm{H}_{2} \mathrm{O}$ and $\mathrm{CO}$ high-temperature opacities derived from

\footnotetext{
${ }^{3}$ Gemini NIR Spectrometer (Elias et al. 2006).
} 
ExoMol (Barber et al. 2006) and HITEMP linelists. Similarly to DENIS J1425-36 and 2MASSJ2208+29, the spectral shape of $H$ and $K$ bands points toward a low-gravity and/or high-metallicity atmosphere which is indeed indicated by the modeled spectrum. The reddening is obtained with an effective $\gamma$ of 1.02 up to 0.1 bars in the atmosphere. Out-ofequilibrium chemistry of $\mathrm{CO} / \mathrm{CH}_{4}$ plays an important role in the atmosphere of PSO J318-22. Unlike DENIS J142536 and 2MASSJ2208+29, that have too high temperatures, PSO J318-22 is sufficiently cold for $\mathrm{CH}_{4}$ to dominate the atmosphere. Out-of-equilibrium processes (that can be linked to fingering convection Tremblin et al. 2016) are crucial to prevent $\mathrm{CH}_{4}$ formation and keep carbon in $\mathrm{CO}$ as expected from the observed spectrum, due to quenching of $\mathrm{CO}$ from the deep atmosphere where it is favoured in chemical equilibrium. In our models, PSO J318-22 is actually right at the transition of having $\mathrm{CH}_{4}$ as the dominant carbon-bearing species. The radius we have obtained is around $\sim 0.13 \mathrm{R}_{\odot}$ which corresponds to an age between 30-50 Myrs, in a good agreement with the estimated age of the Beta Pictoris Moving Group.

\subsection{Color-Magnitude diagram}

Figure 3 shows the CMD $\mathrm{M}_{J}$ versus $J-K$ for field objects and VL-G objects (Liu et al. 2013). The sequence of models has been computed at a fixed surface gravity of $\log g=3.5$, radius of $0.13 \mathrm{R}_{\odot}$, vertical mixing at $10^{6} \mathrm{~cm}^{2} / \mathrm{s}$, effective adiabatic index of $\gamma=1.03$, and with solar metallicity. The range of effective temperature is between $1200 \mathrm{~K}$ and $2000 \mathrm{~K}$, which roughly corresponds to objects of masses between 5 and $20 \mathrm{M}_{\text {jup }}$. As shown in Fig. 1, the effect of gravity and metallicity will be relatively small on the $J$ and $K$ band magnitude, compared to the effect of varying $\gamma$. As expected from the spectral models, this sequence reproduces well the reddening of VL-G objects in the CMD. A sequence with a higher $\gamma$, e.g. 1.05 as for 2MASS J2208+29, would reproduce the field objects with a smaller reddening. Cloud models are currently struggling to reach the reddening of the VL-G objects. Liu et al. (2016) found that the models from Saumon and Marley (2008) and Marley et al. (2012) are systematically too blue. BT-Settl models (Allard et al. 2014) tend to also be bluer than the VL-G objects, and faint red $\mathrm{L}$ dwarfs are not reproduced because the cloud clearing occurs at brighter magnitudes. It is worth stressing that, with the cloud interpretation, these latter models show that there is no correlation between reddening and the $\mathrm{CO} / \mathrm{CH}_{4}$ transition, although observations do show such a correlation both for standard field objects and for VL-G objects.

\section{DISCUSSION AND JWST PERSPECTIVES}

For DENIS J1425-36 and 2MASS J2208+29, the correspondence between the modeled and observed spectra is better in the 1.4-2.4 $\mu \mathrm{m}$ region than below $1.4 \mu \mathrm{m}$. This limitation could be caused by our opacity database that cur- rently contains $\mathrm{TiO}, \mathrm{VO}$, and $\mathrm{FeH}$ but may lack other metal hydrides that could be important at high effective temperatures around $1700 \mathrm{~K}$. As seen in Sect. 2, the spectral shape of the $H$ and $K$ bands clearly indicates a low gravity and/or high metallicity, a degeneracy already observed for other objects (e.g. Delorme et al. 2017). Dedicated studies of the metallicity of moving groups point towards low values, e.g. $[M / H]=0.1$ for $A B$ Doradus (Biazzo et al. 2012). Our models are subject to the same degeneracy and we have generally chosen to keep a metallicity around $[\mathrm{M} / \mathrm{H}]=0.2-0.3$ to be consistent with the metal Galactic distribution (Anders et al. 2017). For these slightly higher metallicities, we still obtain surface gravities $\sim 0.5$ dex lower than expected from evolutionary models (Baraffe et al. 2003). Since these evolutionary models are at solar metallicity, the computation of coherent models at higher metallicity might resolve this discrepancy. Future studies using retrieval analysis will also be useful to better quantify the degeneracy and help assessing if this bias towards high metallicity/low gravity is indeed an issue.

Despite the slightly too-low gravities predicted by our models, the derived radii of the objects are in good agreement with evolutionary models and the ages of the moving groups. Although clouds are predicted to be thicker, and hence easier to detect/characterize in the cloud interpretation, cloudy models have struggled to produce reasonable radii, e.g. in the case of the low-gravity very red HR 8799 planets (see summary in Marley et al. 2012). Therefore, our VL-G models strengthen the interpretation of Tremblin et al. (2016) that NIR reddening is not necessarily a consequence of the presence of clouds near the photosphere, but could be the signature of fingering convection induced by the $\mathrm{CO} / \mathrm{CH}_{4}$ chemical transition.

By using a simple model, we can estimate the magnitude of the velocities that need to be triggered by fingering convection in order to obtain an effective adiabatic index around 1.03. Following Tremblin et al. (2017), we can write the steady state energy conservation equation in the following form:

$$
\begin{aligned}
u_{r, \mathrm{conv}}\left(\nabla_{T}-\frac{\gamma_{\mathrm{ad}}-1}{\gamma_{\mathrm{ad}}}\right) & =\frac{\gamma_{\mathrm{ad}}-1}{\gamma_{\mathrm{ad}}} \frac{H_{\mathrm{rad}}}{\rho g} \\
u_{r, \mathrm{conv}}\left(\nabla_{T}-\frac{\gamma_{\mathrm{eff}}-1}{\gamma_{\mathrm{eff}}}\right) & =0 \\
\frac{\gamma_{\mathrm{eff}}-1}{\gamma_{\mathrm{eff}}} & =\frac{\gamma_{\mathrm{ad}}-1}{\gamma_{\mathrm{ad}}}\left(1+\frac{H_{\mathrm{rad}}}{u_{r, \mathrm{conv}} \rho g}\right)
\end{aligned}
$$

With $\nabla_{T}=\partial \ln T / \partial \ln P, u_{r \text {,conv }}$ the vertical (overturning or fingering) convective velocities, $H_{\text {rad }}$ the radiative heating rate, $\rho$ the density, $g$ the gravity, $\gamma_{\text {ad }}$ the standard adiabatic index of the gas, and $\gamma_{\text {eff }}$ the effective adiabatic index defined by the last equation. In the overturning convective part of the atmosphere, $H_{\mathrm{rad}} \approx 0$ and the atmosphere is adiabatic with $\nabla_{T} \approx\left(\gamma_{\mathrm{ad}}-1\right) / \gamma_{\mathrm{ad}}$. In the fingering convective part, the atmosphere is in a zone where the radiative energy transport cannot be ignore, hence $\nabla_{T} \approx\left(\gamma_{\mathrm{eff}}-1\right) / \gamma_{\mathrm{eff}}$ with $\gamma_{\mathrm{eff}} \neq \gamma_{\mathrm{ad}}$. 


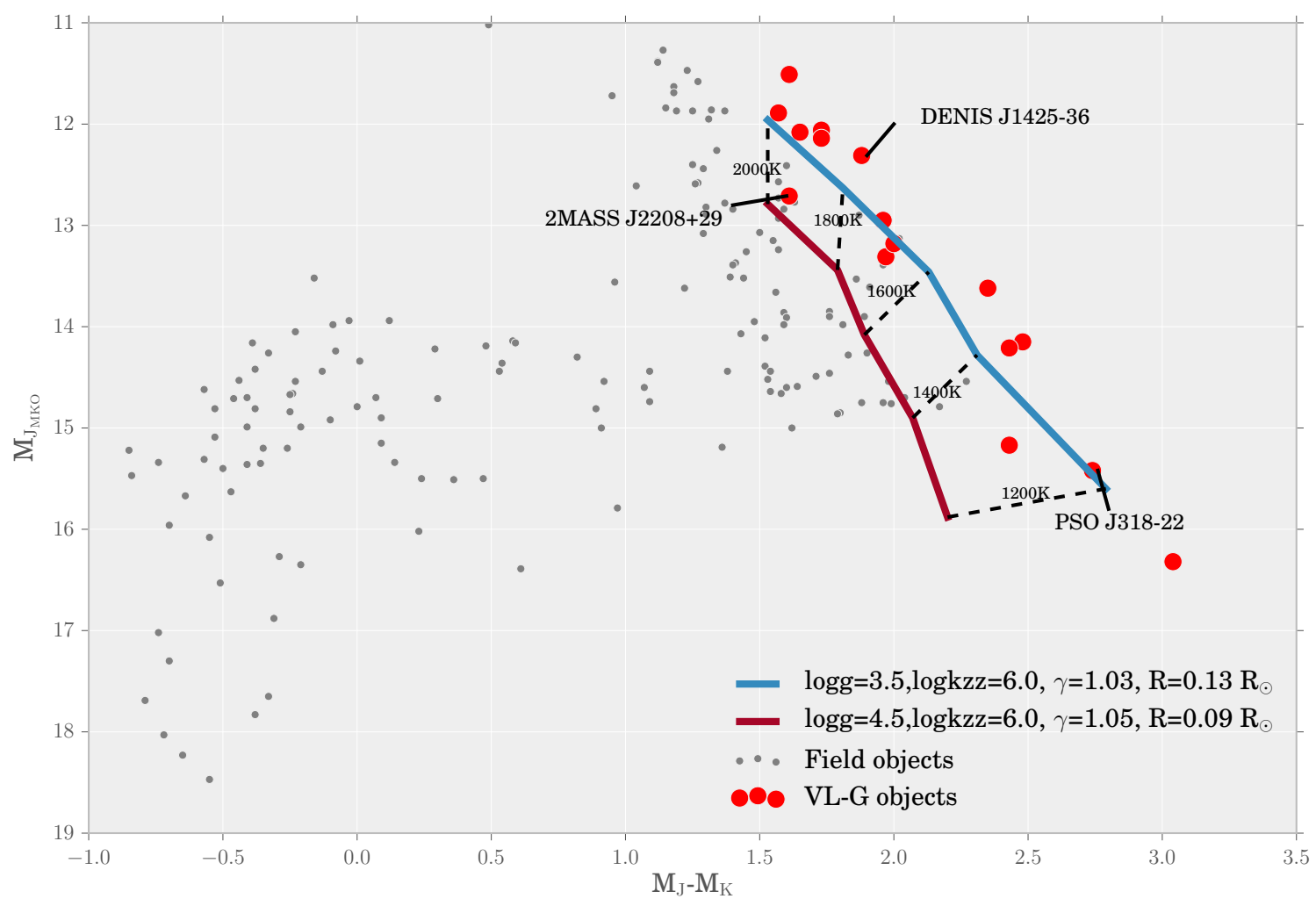

Figure 3. $\mathrm{M}_{J}$ versus $J-K$ MKO color magnitude diagram of field objects (Dupuy and Liu 2012; Faherty et al. 2012), VL-G objects (Liu et al. 2016), and the ATMO model sequence with varying effective temperature.

We can give an order of magnitude estimation of the velocities that are needed to obtain $\gamma_{\mathrm{eff}} \approx 1.03$. For the model of PSOJ318-22, we estimate that fingering-convective velocity of the order of $10 \mathrm{~m} / \mathrm{s}$ are required to obtain such a value of the effective adiabatic index (for comparison velocities in the overturning convection zone are in the range $30-100 \mathrm{~m} / \mathrm{s}$ ).

As in Tremblin et al. (2016), we emphasize that this does not mean that clouds are not present in the atmosphere of these objects. Thin high-altitude clouds are likely to form and be driven to the top of the fingering-convective zone in the atmosphere. They could be observed e.g. in the silicate absorption band at $10 \mu \mathrm{m}$ (Cushing et al. 2006). Although we need to expand the fingering convective zone up to $\sim 0.1$ bar in the atmosphere, it is not clear whether this is sufficient to observe thin high-altitude clouds since the models reach a 10$\mu \mathrm{m}$ optical depth of 1 at $\sim 0.01$ bars. Key future constrains on these signatures will be obtained by JWST with MIRI (see the indicative location of the absorption feature in the MIRI spectral coverage in Fig. 4).

As a conclusion, we recall that fingering convection induced by the $\mathrm{CO} / \mathrm{CH}_{4}$ chemical transition can naturally explain the nature of the $\mathrm{L} / \mathrm{T}$ transition, a transition between fingering-convective "red" CO-dominated atmospheres to stable "blue" $\mathrm{CH}_{4}$-dominated ones. The reddening of the spectrum of field and VL-G objects can be very well explained by a temperature gradient reduction caused by fingering convection. The estimated radii from the models are in good agreement with evolutionary models and the estimated age of moving groups, which strengthens the fingering interpretation.

Nevertheless, as shown in Burningham et al. (2017), both cloud and cloudless models can provide a very good fit to the spectra of field L dwarfs, and cloud models have generally shown good successes in the spectral modelling of field $\mathrm{L}$ and $\mathrm{T}$ dwarfs in the past 20 years. Young low-gravity brown dwarfs could provide a good test to distinguish between the two models if the current limitations of cloudy VL-G models are confirmed by future studies. We provide in Fig. 4 the long wavelength $(3-20 \mu \mathrm{m})$ prediction of our full spectral model for PSO J318-22 compared with a BT-Settl cloudy model and simulated observations with NIRspec using the grating G395M with $\sim 30$ min exposure time. JWST with NIRspec and MIRI observations (SNR $\gtrsim 100$ ) in the 3-7 $\mu \mathrm{m}$ region and future comparisons with cloud models in that wavelength range could help assess which interpretation between clouds and fingering convection is correct. 

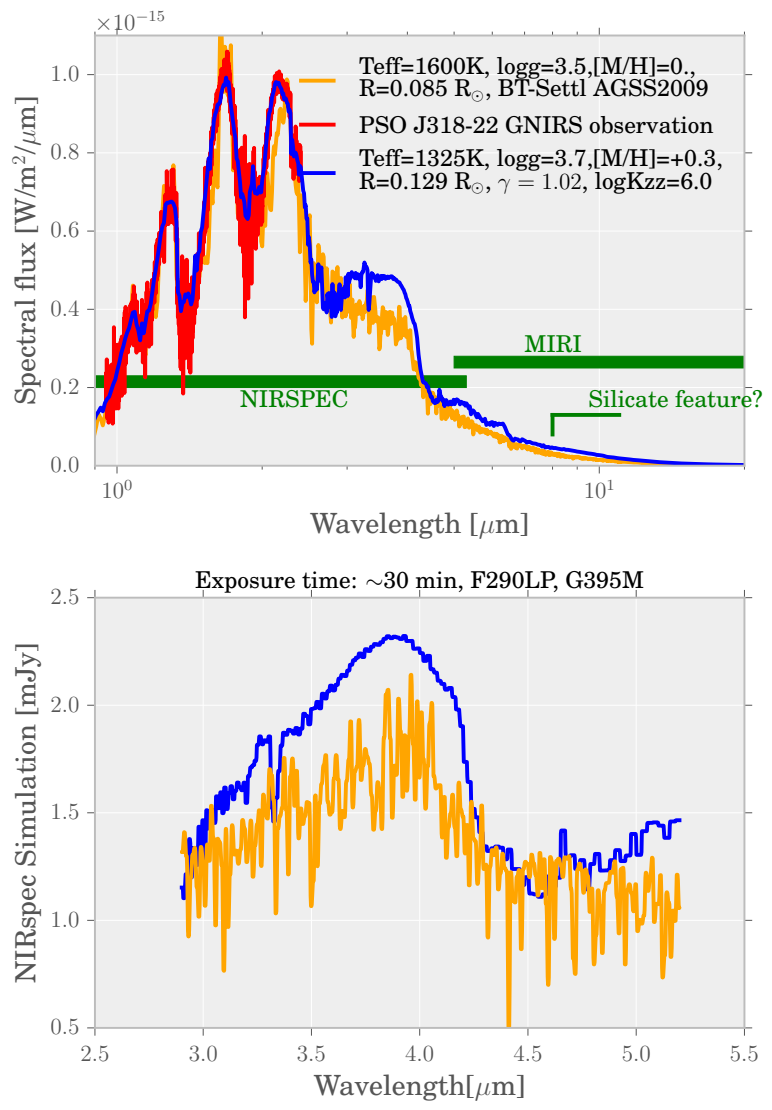

Figure 4. Top: full spectral model of PSO J318-22 with NIRspec/MIRI spectral coverage. Bottom: NIRspec simulation using the grating G395M with a $\sim 30$ min exposure time.
This work is partly supported by the European Research Council under the European Community's Seventh Framework Programme (FP7/2007-2013 Grant Agreement No. 247060-PEPS and grant No. 320478-TOFU). MCL acknowledges support from NSF grant AST-1518339. POL acknowledges support from the LabEx P2IO, the French ANR contract 05-BLAN-NT09-573739.

\section{REFERENCES}

Allard, F., Homeier, D. \& Freytag., B. 2014, ASInC, 11 Buenzli, E., Marley, M. S., Apai, D., et al. 2015, ApJ, 812, 163 Aller, K. M., Liu, M. C., Magnier, E. A., et al. 2016, ApJ, 821, 120 Allers, K. N. \& Liu., M. C. 2013, ApJ, 772, 79

Amundsen, D. S., Baraffe, I., Tremblin, P., et al. 2014, A\&A, 564, 59 Anders, F., Chiappini, C., Minchev, I., et al. 2017, A\&A, 600, A70 Baraffe, I., Chabrier, G., Barman, T. S., et al. 2003, A\&A, 402, 701 R. J. Barber, R. J., Tennyson, J., Harris, G. J., et al. 2006, MNRAS, 368, 1087

Bardalez Gagliuffi, D. C., Burgasser, A. J., Gelino, C. R., et al. 2014, ApJ, 794, 143

Bell, C. P. M., Mamajek, E. E. \& Naylor, T. 2015, MNRAS, 454, 593 Biazzo, K., D'Orazi, V., Desidera, S., et al. 2012 MNRAS, 427, 2905 Burningham, B., Marley, M. S., Line, M. R., et al. 2017, MNRAS, 470, 1177

Burrows, A., Sudarsky, D., \& Hubeny, I. 2006, ApJ, 640, 1063 Chabrier, G. 2002, ApJ, 567, 304

Cushing, M. C., Roellig, T. L., Marley, M. S., et al. 2006, ApJ, 648, 614

Delorme, P., Dupuy, T., Gagné, J., et al. 2017, A\&A, 602, A82

Dieterich, S. B., Henry, T. J., Jao, W.-C., et al. 2014, AJ, 147, 94

Drummond, B., Tremblin, P., Baraffe, I., et al. 2016, A\&A, 594, A69

Dupuy, T. J. \& Liu, M. C. 2012, ApJS, 201, 19

Elias, J. H., Joyce, R. R., Liang, M., et al. 2006, procSPIE, 6269, 62694C
Faherty, J. K., Burgasser, A. J., Walter, F. M., et al. 2012, ApJ, 752, 56 Faherty, J. K., Riedel, A. R., Cruz, K. L. 2016, ApJS, 225, 10 Gagné, J., Lafrenière, D., Doyon, R., et al. 2014, ApJ, 783, 121 Gagné, J., Faherty, J. K., Cruz, K. L., et al. 2015, ApJS, 219, 33 Gagné, J., Lafrenière, D., Doyon, R., et al. 2015, ApJ, 798, 73 Kendall, T. R., Delfosse, X., Martin, E. L., et al. 2004, A\&A, 416, L17 Kirkpatrick, J. D., Reid, I. N., Liebert, J., et al. 2000, ApJ, 120, 447 Kirkpatrick, J. D., Cruz, K. L., Barman, T. S., et al. 2008, ApJ, 689, 1295 Hindmarsh, A. C., 1983, IMACS Transactions on Scientific Computation, 1,55

Liu, M. C., Magnier, E. A., Deacon, N. R., et al. 2013, ApJL, 777, L20 Liu, M. C., Dupuy, T. J. \& Allers, K. N. 2016, ApJ, 833, 96 Marley, M. S., Saumon, D., Cushing, M. 2012, ApJ, 754, 135 Rothman, L., Gordon, I., Barber, R., et al. 2010, JQSRT, 111, 2139 Saumon, D. \& Marley, M. S. 2008, ApJ, 689, 1327

Tennyson, J. \& Yurchenko, S. N. 2012, MNRAS, 425, 21 Tremblin, P., Amundsen, D. S., Mourier, P., et al. 2015, ApJL, 804, L17 Tremblin, P., Amundsen, D. S., Chabrier, G. 2016, ApJL, 817, L19 Tremblin, P., Chabrier, G., Mayne, N. J., et al. 2017, ApJ, 604, A79 Venot, O., Hébrard, E., Agúndez, M., et al. 2012, A\&A, 546, A43 